\title{
La enseñanza monumentalista y utilitarista de las causas de la biodiversidad y de las estrategias para su conservación: un estudio sobre la transposición didáctica de los manuales de la Educación Secundaria española
}

\author{
The monumentalist and utilitarian teaching of the causes of \\ biodiversity and conservation strategies: a study on the didactic \\ transposition of Spanish Secondary Education textbooks
}

\author{
Gonzalo Miguel Angel Bermudez ${ }^{1}$ • Ana Lía De Longhi ${ }^{1}$ • \\ Valentín Gavidia ${ }^{2}$
}

\begin{abstract}
Resumen: Este trabajo estudia la presencia y complejidad en el tratamiento de las causas de la biodiversidad y de las razones para su conservación que realizan manuales escolares de la Educación Secundaria de España. Para ello, se determinó el ámbito conceptual y axiológico que define la comunidad de expertos, se examinó el tratamiento propuesto en el currículo español y se analizaron 50 manuales escolares (27 de la Educación Secundaria Obligatoria -ESO - y 30 del Bachillerato). Los resultados mostraron que el proceso de transposición didáctica vuelve monumentalista la enseñanza de la biodiversidad, ya que olvida las razones de su existencia, y se enriquece de connotaciones biologicistas y utilitaristas sobre los motivos de su conservación, dejando de lado las dimensiones éticas, filosóficas y culturales. Además, el tratamiento de los manuales escolares de las causas y conservación de la biodiversidad reveló una marcada distancia de lo señalado en el currículum de España.
\end{abstract}

Palabras-clave: Diversidad biológica. Enseñanza de la biología. Chevallard. Libro de texto. Parques nacionales.

\begin{abstract}
This paper studies the presence and complexity of the treatment of the causes of biodiversity and the reasons for its conservation in Spanish secondary education textbooks. To this purpose, a conceptual and axiological framework defined by an expert community was reviewed, the treatment proposed in the Spanish national curriculum was described and a total of 50 textbooks were analyzed (27 from middle and 30 from high schools). The results showed that the process of didactic transposition makes the teaching of biodiversity monumental, since it omits the reasons for its existence. Consequently, teaching becomes entrenched in biologicist and utilitarian connotations about the motives for its conservation, thereby leaving aside the ethical, philosophical and cultural dimensions. Furthermore, the treatment of the causes and reasons for biodiversity conservation in textbooks revealed a marked distance from the curriculum of Spain, especially for the first year of middle and high school.
\end{abstract}

Keywords: Biodiversity. Biology teaching. Chevallard. Textbook. National parks.

\footnotetext{
${ }^{1}$ Departamento de Enseñanza de la Ciencia y la Tecnología, Universidad Nacional de Córdoba (UNC), Avenida Vélez Sársfield 1611, X5016GCA, Córdoba, Argentina. E-mail: <gbermudez@com.uncor.edu>

${ }^{2}$ Departamento de Didáctica de las Ciencias Experimentales y Sociales, Universidad de Valencia, Valencia, España.
} 


\section{Introducción}

La "transposición didáctica" es el fenómeno por el cual el conocimiento científico se transforma para ser enseñado. Para Chevallard (1991), el conjunto de pasos por el que un objeto de saber se transforma en objeto de enseñanza se denomina transposición didáctica en sentido amplio (lato sensu), mientras que la transformación de un objeto a enseñar a objeto de enseñanza se corresponde con la transposición en sentido estricto (stricto sensu). Como resultado de estos procesos, el conocimiento asume modalidades y funciones diferentes a las del saber científico, ya que está sometido a diferentes exigencias de productividad (CHEVALLARD, 1991). En este sentido, Verret (1975) explica que son tres las etapas de la transposición que pueden conducir a un conocimiento exiliado de su origen social e histórico: (a) desincretización, cuando se delimitan parcelas de saber descontextualizadas de las problemáticas que originalmente le dan sentido (como su anclaje social, su corpus teórico e implicancias metodológicas, etc.); (b) despersonalización, la publicación del saber permite que sea interpretado, reproducido y aplicado por otros; y (c) programabilidad, ya que se determina una progresión en la adquisición del saber, definiendo el orden en el que se estudian los distintos temas. En este último punto adquiere gran importancia la textualización debido a que el saber público ofrece una variable de control muy sensible y sobre la cual la instancia política tiene asegurado el "control” por medio de los programas, lineamientos oficiales y manuales escolares (CHEVALLARD, 1991). Estos materiales legitiman y justifican el proyecto social al que responde el sistema educativo. Por otro lado, los procesos de desincretización y despersonalización pueden llevar a la monumentalización de los saberes (CHEVALLARD, 2004) cuando el conocimiento escolar es presentado como una obra terminada, valiosa per se, perdiendo de este modo su carácter funcional.

Para Chevallard (1991), el sistema educativo está integrado por una esfera de personas que "piensan sobre la educación" e integran la noosfera; es decir, padres, órganos políticos educativos, asociaciones docentes y especialistas de las distintas disciplinas. Este sistema está en equilibrio con la noosfera si se cumplen dos condiciones: primero, que el saber enseñado sea visto por los científicos como suficientemente cercano al saber sabio, hecho que legitima el proceso educativo como proyecto social; y, segundo, que el saber enseñado a los estudiantes esté alejado de lo que saben sus padres. Cuando lo anterior no ocurre, el saber se desgasta en un sentido biológico, ya que lo que se enseña es viejo en relación a la ciencia, y moral, debido a que no representa un cambio frente a las generaciones precedentes.

El trabajo con la transposición resulta clave para el didacta ya que "permite recapacitar, tomar distancia, [...] poner en cuestión las ideas simples, desprenderse de la familiaridad engañosa de su objeto de estudio. En una palabra, lo que permite ejercer su vigilancia epistemológica" (CHEVALLARD, 1991, p. 16). En los últimos años, las investigaciones sobre transposición han tomado a los libros de texto de distintas disciplinas como material de estudio privilegiado. Sin embargo, debemos reconocer que el fenómeno de transposición no debe reducirse a los contenidos presentes en éstos sino que también deberían considerarse los conocimientos que se construyen en las mismas aulas (BOSCH; GASCÓN, 2006). Para la enseñanza de la Biología, los estudios sobre manuales escolares han puesto énfasis en la descripción de errores para temas de genética molecular (MARTÍNEZ-GRACIA; GIL-QUÍLEZ; OSADA, 2006), de salud (ASSIS; PIMENTA; SCHALL, 2013), de evolución biológica (ALMEIDA; FALCÃO; ROCHA, 2010), y en las características de las ilustraciones sobre determinados procesos (CAMPOS; LIMA, 
2008; MALDONADO GONZÁLEZ; GONZÁLEZ GARCÍA; JIMÉNEZ TEJADA, 2007). También han sido investigadas las tareas indicadas para los alumnos, tales como las prácticas de argumentación y de experimentación en el laboratorio (ALMEIDA; FIGUEIREDO; GALVÃO, 2012; SELLES; FERREIRA, 2010).

En cuando a la diversidad biológica, su pérdida es considerada uno de los problemas ambientales más urgentes a escala global en el ámbito científico y político (CONVENTION ON BIOLOGICAL DIVERSITY, 2003). La Convención sobre la Diversidad Biológica (CBD) de 1992, definió a la biodiversidad como aquella constituida por todos los organismos terrestres y acuáticos (incluyendo los animales, las plantas y los microbios) a todas las escalas; es decir, desde la diversidad genética dentro de las poblaciones, a la diversidad de especies, a la diversidad de comunidades a lo largo de los paisajes (CONVENTION ON BIOLOGICAL DIVERSITY, 1992). A pesar de estos desarrollos, han sido descritos numerosos problemas en el tratamiento que realizan los libros de texto de la educación secundaria sobre la diversidad biológica; por ejemplo, la descontextualización del ámbito axiológico y social (MARTÍNEZ BERNAT; GARCÍA GÓMEZ, 2009), el centrismo en las especies y su riqueza como único componente y atributo de la biodiversidad (BERMUDEZ et al., 2014; BERMUDEZ; DE LONGHI, 2012), el desarrollo de más contenidos y de forma más llamativa para especies animales que para vegetales (RODRÍGUEZ MIRANDA et al., 2014), y la presentación de conceptos de modo superficial o con errores conceptuales (CARDOSO-SILVA; OLIVEIRA, 2013). En la actualidad, la noción de biodiversidad emerge en la concepción de un nuevo paradigma, que se aleja del determinismo biológico, y que plantea la necesidad de su estudio de modo holístico y estructurante de la enseñanza (GUTIÉRREZ HERNÁNDEZ, 2013).

Los objetivos de investigación del presente trabajo fueron: (a) elaborar un instrumento de análisis de las causas de la existencia y de las estrategias de conservación de la biodiversidad que sea factible de ser aplicada a manuales escolares de la educación secundaria, y (b) analizar la transposición didáctica que se promueve en los libros de texto del bachillerato y de la educación secundaria obligatoria (ESO) de España, estableciendo niveles de complejidad en el tratamiento del contenido.

\section{Materiales y Métodos}

En una primera fase se determinó el ámbito conceptual y axiológico de las causas de la biodiversidad y su conservación, el cual es definido por la comunidad de expertos a partir de la lectura de literatura especializada. Se construyó una matriz de análisis por categorías y subcategorías, las que fueron cotejadas con una muestra de manuales escolares. Por razones de espacio acotado, la matriz se presenta acompañada de los resultados en las Tablas 1 y 2. Luego, en la segunda etapa se analizó el programa nacional de estudios propuesto por el Ministerio de Educación de España con el fin de determinar en qué espacios curriculares del bachillerato y de la ESO tiene presencia la biodiversidad como tema general, sus causas y conservación en particular. Por último, la tercera fase de esta investigación comprendió el estudio de los textos propiamente dichos, con base en manuales publicados desde 1995, periodo que comprende dos legislaciones educativas españolas: Ley Orgánica General del Sistema Educativo (LOGSE) (ESPAÑA, 1991) y la Ley Orgánica de Educación (LOE) (ESPAÑA, 2007b). El listado 
completo de manuales $(n=50)$ se presenta en el Anexo A, e indica que cada material recibió un código dado por su nivel educativo: ESO para la Educación Secundaria Obligatoria $(n=27)$ y BAC para el bachillerato $(n=33)$. Además, los manuales fueron clasificados por el curso del nivel educativo correspondiente; es decir, " 1 ” ( $n=13)$ para libros de primero de la ESO (Ciencias de la Naturaleza), y “4” (n=14) para libros de cuarto de la ESO (Biología y Geología). Para libros del bachillerato, la codificación fue "1" ( $n=13$; para libros de Biología I, de Biología y Geología) y "2" ( $n=10)$, para libros de Ciencias de la Tierra y Medioambientales $(n=10)$. En todos los casos la variable de respuesta fue la presencia o ausencia del tratamiento del contenido especificado en cada categoría y sub-categoría de análisis. Además, se transcribieron textualmente las citas para ejemplificar e interpretar cualitativamente el tratamiento de la biodiversidad.

Los datos categóricos (presencia/ausencia) se analizaron mediante la prueba de Chi-cuadrado $\left(\chi^{2}\right)$ con el programa estadístico SPSS ${ }^{\circ}$ (Statistical Package for Social Sciences, versión $17.0)^{3}$, se trabajó con un nivel de significancia de $\alpha=0.05$, y los resultados se expresaron como porcentajes. También se realizaron pruebas estadísticas multivariadas como el análisis de conglomerados (o "cluster") y el análisis de componentes principales (ACP). El agrupamiento de objetos multivariados (conglomerados) es frecuentemente utilizado para obtener mayor conocimiento sobre la estructura de las observaciones y variables en estudio. En tanto, el ACP encuentra un nuevo conjunto de variables (componentes principales), no correlacionadas entre sí, que explican la estructura de variación de los casos observados con una mínima pérdida de información (BALZARINI et al., 2008). El sistema de coordenadas que explica la mayor varianza del conjunto de datos se denomina componente 1 , mientras que la segunda coordenada, componente 2, y así sucesivamente. La proporción de variabilidad total explicada por estas componentes (o autovalor) se expresa como porcentaje, logrando reducir las dimensiones de los datos a dos o más ejes cartesianos. En los gráficos resultantes, las variables se grafican como vectores desde el origen (intersección entre componentes), mientras que las observaciones (manuales escolares) se identifican como puntos. En el ACP, la distancia entre las observaciones y las variables carece de interpretación, a diferencia de las direcciones de éstas desde el origen. De este modo, los ángulos agudos y obtusos entre vectores pueden ser interpretados como correlaciones entre variables, mientras que ángulos de $90^{\circ}$ indican que son independientes (BALZARINI et al., 2008). Para realizar estos análisis, se utilizó el programa Infostat (Universidad Nacional de Córdoba, versión 2013) ${ }^{4}$, y las variables categóricas de respuesta fueron transformadas en ceros (ausencia) y unos (presencia).

\footnotetext{
${ }^{3}$ Disponible en: <http://www-01.ibm.com/software/analytics/spss/>. Acceso el: 26 mayo 2015.

${ }^{4}$ Disponible en: < http://www.infostat.com.ar/>. Acceso el: 26 mayo 2015.
} 


\section{Resultados y discusión}

\section{Las causas de la biodiversidad y las estrategias para su conservación en el currículo de España}

\section{a) Educación Secundaria Obligatoria (ESO)}

En el primer curso de la ESO, la biodiversidad es tratada en el espacio curricular "Ciencias de la naturaleza", dentro del bloque "Los seres vivos y su diversidad", donde se estudia la clasificación de los seres vivos en reinos y la "valoración de la importancia de mantener la diversidad de los seres vivos. Análisis de los problemas asociados a su pérdida” (ESPAÑ, 2007b, p. 694). Ciertos aspectos relacionados con las causas de la biodiversidad se presentan en el bloque 3 ("Materiales terrestres"), estudiando el clima y el papel protector de la atmósfera para los seres vivos. En el segundo curso de la LOE y la LOGSE, la biodiversidad se estudia indirectamente en el bloque 5 ("La vida en acción”) y 3 ("La energía y los seres vivos”) de la materia "Ciencias de la naturaleza", respectivamente, a través de los componentes de los ecosistemas y las relaciones entre los factores bióticos y abióticos. Por otro lado, en el tercer curso de la LOE comienza a separarse la Biología y la Geología de la Física y la Química como núcleos temáticos. El bloque 6 ("Las personas y el medio ambiente") de "Biología y Geología" desarrolla problemáticas ambientales como la contaminación del aire y del recurso hídrico, y la gestión de los residuos, pero no se considera la pérdida de la biodiversidad como un problema actual ni para la "valoración de la necesidad de cuidar del medio ambiente y adoptar conductas solidarias y respetuosas con él” (ESPAÑA, 2007b, p. 697). A pesar de que se propone como criterio de evaluación del tercer curso "recopilar información procedente de diversas fuentes documentales acerca de la influencia de las actuaciones humanas sobre los ecosistemas: efectos de la contaminación, desertización, disminución de la capa de ozono, agotamiento de recursos y extinción de especies" (ESPAÑA, 2007b, p. 698), no se explicitan la biodiversidad ni las estrategias para su conservación. En el cuarto curso de la ESO, la Biología y la Geología consolidan su separación de las otras ciencias de la naturaleza en un espacio curricular propio. Para este curso, el bloque "Evolución de la vida" de la materia "Biología y Geología" propone la "valoración de la biodiversidad como resultado del proceso evolutivo. El papel de la humanidad en la extinción de las especies y sus causas" (ESPAÑA, 2007b, p. 701). En relación con las causas de la biodiversidad, también se estudia la aparición y la extinción de las especies. En tanto, el bloque 4 ("Las transformaciones en los ecosistemas") trata las interacciones alimentarias en el ecosistema: "identificación de cadenas y redes tróficas en ecosistemas terrestres y acuáticos” (ESPAÑA, 2007b, p. 701) y los disturbios ambientales, analizando sus efectos en las sucesiones ecológicas.

\section{b) Bachillerato}

En el bachillerato de la LOE (ESPAÑA, 2007a), la biodiversidad se trabaja principalmente en la materia "Ciencias para el mundo contemporáneo", la que estuvo ausente del programa de la ley anterior (Real Decreto 1178/1992). Este espacio curricular común a todos los itinerarios del bachillerato se originó para responder a la demanda de que las ciencias debían formar parte de la cultura general. En esta asignatura, la biodiversidad se ubica en el bloque "Hacia una gestión sostenible del planeta", estudiando "La contaminación, la desertización, el aumento de residuos y la pérdida de biodiversidad. El cambio climático” (ESPAÑA, 2007a, p. 
45388). Para la modalidad "Ciencias y Tecnología” de la LOE, también para el primer curso del bachillerato, el espacio curricular "Biología y Geología” profundiza sobre los contenidos sobre taxonomía desarrollados en la misma materia de la ESO. Por ejemplo, el bloque 4 "Unidad y diversidad de la vida" incluye el contenido "La diversidad de los seres vivos y el problema de su clasificación. Criterios de clasificación” (ESPAÑA, 2007a, p. 45435). Asimismo, en los bloques 5 y 6 se analiza detalladamente la diversidad de plantas y animales, respectivamente (en correspondencia con los temas 5, 7 y 8 de la LOGSE). Cabe destacar que el bloque "La biología de los animales" trata la importancia de la diversidad animal, los animales en peligro de extinción y las acciones para la conservación de la diversidad.

En el segundo, y último, curso del bachillerato de la LOE, la materia "Biología" tiene una mirada molecular y subcelular que "pretende ofrecer una visión actualizada de la materia [...], ampliar y profundizar los conocimientos científicos sobre los mecanismos básicos que rigen el mundo vivo, para lo cual es necesario tratar los niveles celular, subcelular y molecular [...]" (ESPAÑA, 2007a, p. 45431). El único tema relacionado con la biodiversidad para esta asignatura se corresponde con el "estudio de la diversidad de microorganismos. Sus formas de vida. [...]. Interacciones con otros seres vivos. Intervención de los microorganismos en los ciclos biogeoquímicos" (ESPAÑA, 2007a, p. 45432). En cambio, se recuperan las temáticas ambientales y la conceptualización de la diversidad biológica en la asignatura "Ciencias de la Tierra y Medioambientales” del segundo curso de la LOE (ESPAÑA, 2007a, p. 45436), la que se plantea como "una ciencia de síntesis y de aplicación de otras ciencias, entre las que figuran destacadamente la geología, la biología, la ecología, la química y la física, junto con otras aportaciones procedentes del campo de las ciencias sociales". El término "biodiversidad" figura en el bloque 4 ("La ecosfera") al tratar "La biosfera como patrimonio y como recurso frágil y limitado. Impactos sobre la biosfera: deforestación y pérdida de la biodiversidad" (ESPAÑA, 2007a, p. 45437). También se estudia el paso del tiempo en los ecosistemas (sucesión ecológica, autorregulación y regresión) y las relaciones tróficas entre los organismos. En el caso del currículo de la LOGSE, estos conceptos se desarrollan en el tema 7 "La ecosfera: ecosfera, biosfera y ecosistema" con un tratamiento temático semejante. Por último, el bloque 6 ("La gestión del planeta") de la LOE plantea el tratamiento del marco legal para la conservación de la naturaleza en el apartado "Legislación medioambiental. La protección de espacios naturales" (ESPAÑA, 2007a, p. 45438).

\section{Análisis de los manuales escolares}

\section{a) Causas de la diversidad biológica}

Las causas de la biodiversidad fueron escasamente tratadas en los libros de texto de ambos niveles educativos, sin diferencias significativas entre éstos para la mayoría de las categorías estudiadas (Tabla 1). El régimen de disturbios fue la causa más frecuentemente encontrada en los manuales de la ESO (51.9\%). Para este caso, la mayoría de las relaciones conceptuales se establecieron a partir de reconocer los cambios en el tiempo de un ambiente, lo que se denomina sucesión ecológica. Analizando el tratamiento de este fenómeno, Ibarra Murillo y Gil Quílez (2005) concluyeron que el proceso de transposición se realiza en los libros a través de una simplificación de la teoría de la sucesión desde el modelo holístico, sumado a la polisemia de muchas de las nociones empleadas para explicarla. En este sentido, en el presente estudio se encontró que la 
transposición de las causas de la biodiversidad estuvo mediada por las nociones de estabilidad, equilibrio y una excesiva simplificación en el tratamiento de la sucesión ecológica. Por otro lado, se encontraron diferencias significativas entre los cursos de la educación secundaria, con una amplia presencia del tratamiento de los disturbios en el segundo curso del bachillerato y en el cuarto curso de la ESO (Tabla 1).

Como ejemplo del cuarto curso de la ESO, se cita un manual en el que se relacionó la biodiversidad con las etapas de la sucesión y la partición del nicho ecológico: "En las últimas etapas de la sucesión aparece un incremento de la diversidad. [...] aumenta la biodiversidad (número de especies), el espacio se subdivide en un elevado número de nichos ecológicos y aumenta el número de interrelaciones" (ESO4M, p. 92). En tanto, para el primer curso del bachillerato, el efecto de los disturbios en la biodiversidad fue considerado negativamente, por ejemplo para el manual BAC1M, ya que refirió a que el ritmo gradual del crecimiento de España, en relación a otros países de la zona, ha propiciado una gran diversidad biológica (p. 147). En cambio, en el segundo curso del bachillerato se incorporaron a los elementos anteriores los conceptos de niveles tróficos y se resignificó la noción de nicho ecológico:

Aumento de la diversidad de especies, especialmente en la comunidad clímax, en relación con las fases iniciales, así como mayor complejidad en la estructura del ecosistema en sus etapas finales: el número de niveles tróficos y de interacciones por el alimento entre las diversas especies es máximo. [...] En el clímax hay ausencia de nichos sin explotar. (BAC2F, p. 266)

Tabla 1. Distribución de frecuencias (en porcentaje) para la categoría de análisis "causas de la existencia de la diversidad biológica" en manuales de la ESO y del bachillerato

\begin{tabular}{|c|c|c|c|c|c|c|c|c|c|}
\hline \multirow[t]{2}{*}{ I. Causas } & \multicolumn{3}{|c|}{ Nivel } & \multicolumn{5}{|c|}{ Curso } & \multirow[b]{2}{*}{ Total } \\
\hline & ESO & BAC & $\overline{\chi^{2}}$ & ESO1 & ESO4 & BAC1 & BAC2 & $\chi^{2}$ & \\
\hline 1. Variables climáticas & 33.3 & 17.4 & ns & 38.5 & 28.6 & 7.7 & 30.0 & ns & 26.0 \\
\hline 2. Régimen de disturbios & 51.9 & 30.4 & ns & 0.0 & 100.0 & 7.7 & 60.0 & $* *$ & 42.0 \\
\hline 3. Disponibilidad de recursos & 0.0 & 8.7 & ns & 0.0 & 0.0 & 0.0 & 20.0 & $* a$ & 4.0 \\
\hline 4. Interacciones entre especies & 11.1 & 21.7 & ns & 0.0 & 21.4 & 7.7 & 40.0 & *a & 16.0 \\
\hline 5. Otras (latitud, altitud, etc.) & 22.2 & 56.5 & $*$ & 30.8 & 14.3 & 23.1 & 100.0 & $* * \mathrm{a}$ & 38.0 \\
\hline
\end{tabular}

$\mathrm{BAC}=$ bachillerato, $\mathrm{ESO}=$ educación secundaria obligatoria, 1,2 y $4=$ años del nivel educativo correspondiente. ${ }^{*}$ Significativo con $\mathrm{p} \leq 0.05,{ }^{* *}$ significativo con $\mathrm{p} \leq 0.001$. ns $=$ no significativo. ${ }^{a}=\geq 25 \%$ de las casillas poseen una frecuencia esperada inferior a 5.

Fuente: Elaborado por los autores.

Por otro lado, los manuales escolares también relacionaron la biodiversidad con la ubicación geográfica (latitud) y la variedad de hábitats, factores presentes en el $56.5 \%$ de los manuales del bachillerato (100\% del segundo curso), frente a un $22.2 \%$ de los manuales de la ESO (Tabla 
1). Por ejemplo, algunos de los textos del primer curso de la ESO explicaron la alta riqueza de especies en la zona ecuatorial y en España por la latitud y la variedad de paisajes: "La situación geográfica de nuestro país y su variedad de climas y paisajes han propiciado el desarrollo de una gran diversidad biológica, comparable a la de los bosques tropicales mejor conservados" (ESO1H, p. 146). En el cuarto curso de la ESO se repitieron las nociones anteriores y se incluyeron los conceptos de movimientos del eje de la Tierra: "La distribución de la vida en nuestro planeta se deriva del hecho de que la energía solar que recibe la superficie terrestre no es uniforme [lo que] [...] origina las distintas zonas climáticas de la Tierra con sus comunidades correspondientes" (ESO4E, p. 199) y de endemismo: "La gran diversidad de climas y de paisajes de nuestro territorio [...] se traduce en la diversidad biogeográfica y en un número elevado de endemismos presente en la naturaleza española" (ESO4F, p. 134).

En el segundo curso del bachillerato se mantuvo el concepto de nicho ecológico y se incorporó el de especiación: "A mayor heterogeneidad espacial de territorio es mayor el número de especies. La presencia de zonas reducidas de características marcadamente distintas del resto favorece la especiación alopátrica” (BAC2G, p. 226). Siguiendo el mismo patrón de resultados, los libros del segundo curso del bachillerato y del cuarto curso de la ESO fueron los que consideraron a las interacciones entre especies (competencia, depredación, etc.) como uno de los factores que regulan la biodiversidad: "La competencia puede ser un factor importante para generar una elevada diversidad [ya que] [...] puede ser mayor en entornos favorables, donde los organismos pueden especializarse y tener nichos restringidos". (BAC2G, p. 226).

\section{b) Conservación de la biodiversidad}

\section{i. Razones}

Según Martínez Bernat y García Gómez (2009), los manuales españoles muestran una desconexión de la biodiversidad con sus dimensiones axiológicas y sociales. De acuerdo con estos autores, los textos de la ESO estudiados en el presente trabajo desarrollaron escasamente las razones para conservar la biodiversidad, encontrando que sólo tres libros mencionaron motivaciones estéticas, biológicas y ético-filosóficas (Tabla 2). Si se consideran ambos niveles de la educación secundaria, las razones de utilidad fueron las más frecuentemente citadas, asociándolas a los bienes y servicios ecosistémicos que aporta la naturaleza (BERMUDEZ et al. ${ }^{5}$; MENZEL; BÖGEHOLZ, 2009). Por ejemplo, un manual del primer curso de la ESO señala que:

La biodiversidad es un recurso muy valioso. Nos proporciona alimentos, medicamentos, energía, materias primas y entornos de gran belleza para nuestro goce. [...] Obtenemos alimentos básicos de las plantas, de la ganadería y de la pesca. [...] Los bosques nos proporcionan numerosas materias primas como ahora el suru, el caucho, la celulosa o la fusta [...]. (ESO1E, p. 178)

\footnotetext{
${ }^{5}$ Datos no publicados.
} 
Por otro lado, en el cuarto curso de la ESO, las razones utilitarias de conservación de la biodiversidad se presentan en un porcentaje menor que los manuales del primer curso $(21.4 \%)$, aunque de forma indirecta: "La pérdida de especies animales y vegetales puede generar a largo plazo grandes problemas ambientales. [...]. Además, esas especies que se extinguen podrían ser el origen de nuevos productos y medicamentos [...]" (ESO4J, p. 51). A diferencia de los manuales de este nivel educativo, la totalidad de los textos del segundo curso del bachillerato aportó argumentos sobre la utilidad de la biodiversidad. Una de las razones más complejas incluyó la distribución de los usos económicos de la biodiversidad, dándole un contexto social a la perspectiva utilitaria:

La biodiversidad se convierte en un nuevo factor de enfrentamiento geopolítico: la mayoría de los recursos genéticos se encuentran en el Sur, en los bosques tropicales, mientras que la mayor parte de las industrias usuarias están en el Norte. Los países del Sur rechazan que, al declararlos reserva de biodiversidad, se les limite su industrialización [… (BAC2A, p. 95)

Por otro lado, las razones estéticas para conservar la diversidad biológica fueron más citadas en los manuales del bachillerato que en los de la ESO, pero en ambos casos la belleza fue el único valor: "La biodiversidad afecta a la vida humana de muchas maneras. Hay importantes dimensiones estéticas y éticas, pero, además, parte de nuestra existencia depende de su uso" (BAC1G, p. 194). Las raz̧ones ético-filosóficas también fueron más nombradas en los manuales del bachillerato que en los de la ESO, aunque sin diferencias significativas:

Las especies, desde un punto de vista ético, tienen derecho a vivir en su medio natural; y desde el punto de vista de la cultura ecológica, cada especie es una obra de arte de la naturaleza. De igual modo que nadie espera obtener beneficios de Las Meninas de Velázquez y, sin embargo, todos estamos de acuerdo en que sería una tragedia su destrucción, se debería comprender que los linces o la cigüeña negra, son una obra de arte irrepetible de la naturaleza. (BAC2C, p. 398)

En coincidencia con nuestros resultados, Martínez Bernat y García Gómez (2009) pusieron de manifiesto que los manuales del cuarto curso de la ESO mostraron un pronunciado desbalance en el tratamiento de conceptos relevantes para la interpretación de acciones de conservación de la biodiversidad. Esta situación tiene lugar en el marco de una radical descontextualización en el desarrollo de la temática de la biodiversidad del ámbito ético y político-social (GARCÍA GÓMEZ; MARTÍNEZ BERNAT, 2010).

\section{ii. Estrategias}

La red Natura 2000 constituye uno de los proyectos más ambiciosos para detener la pérdida de la biodiversidad y la degradación de los servicios ecosistémicos en la Unión Europea (EUROPEAN PARLIAMENT, 2011), y por la cual se designan sitios de conservación de 
acuerdo con criterios ecológicos y biogeográficos. Esta red incluye las Zonas de Protección para las Aves (ZEPA), establecidas en virtud de la Directiva Aves y caracterizadas por ser áreas naturales de singular relevancia para la conservación de la fauna avícola (COUNCIL OF THE EUROPEAN COMMUNITIES, 1979 y modificatorias; EUROPEAN COMMISSION, 2014). En la actualidad, el $8.8 \%$ del territorio español está protegido por alguna normativa y más del $25 \%$ del país se halla incluido en la red Natura 2000 (CONVENTION ON BIOLOGICAL DIVERSITY, 2015).

En los manuales escolares estudiados, la estrategia de conservación de la biodiversidad mayormente tratada fue la creación de parques nacionales y reservas, lo que se conoce como conservación in-situ (Tabla 2). Martínez Bernat y García Gómez (2009) señalan la importancia de comprender conceptos como el de la sostenibilidad, red Natura 2000 y las zonas ZEPA para que el ciudadano sea capaz de interpretar noticias ambientales. A pesar de ello, sólo se encontraron estrategias de conservación en el $23.1 \%$ de los manuales del primer curso de la ESO y del bachillerato.

Tabla 2. Distribución de frecuencias (en porcentaje) para la categoría de análisis "razones, estrategias y aspectos legales de la conservación de la diversidad biológica" en manuales de la ESO y del bachillerato español

\begin{tabular}{|c|c|c|c|c|c|c|c|c|c|}
\hline \multirow{2}{*}{$\begin{array}{l}\text { Categorías } \\
\text { de análisis }\end{array}$} & \multicolumn{3}{|c|}{ Nivel } & \multicolumn{5}{|c|}{ Curso } & \multirow[b]{2}{*}{ Total } \\
\hline & ESO & BAC & $\overline{\chi^{2}}$ & ESO1 & ESO4 & BAC1 & BAC2 & $\chi^{2}$ & \\
\hline \multicolumn{10}{|l|}{ I. Razones } \\
\hline 1. De utilidad/económicas & 29.6 & 56.5 & ns & 38.5 & 21.4 & 23.1 & 100.0 & $* * *$ & 42.0 \\
\hline 2. Estéticas & 11.1 & 34.8 & $*$ & 15.4 & 7.1 & 15.4 & 60.0 & $* * a$ & 22.0 \\
\hline 3. Biológicas & 11.1 & 34.8 & $*$ & 7.7 & 14.3 & 23.1 & 50.0 & ns & 22.0 \\
\hline 4. Éticas/Filosóficas & 11.1 & 13.0 & ns & 15.4 & 7.1 & 15.4 & 10.0 & ns & 12.0 \\
\hline \multicolumn{10}{|l|}{ II. Estrategias } \\
\hline 1. Parques, reservas, otros & 33.3 & 52.2 & ns & 23.1 & 42.9 & 23.1 & 90.0 & $* *$ & 42.0 \\
\hline 2. Comercio de flora y fauna & 14.8 & 30.4 & ns & 15.4 & 14.3 & 7.7 & 60.0 & $* a$ & 22.0 \\
\hline \multicolumn{10}{|l|}{ III. Aspectos legales } \\
\hline $\begin{array}{l}\text { 1. Tratados, reuniones, cumbres } \\
\text { internacionales }\end{array}$ & 29.6 & 52.2 & ns & 30.8 & 28.6 & 15.4 & 100.0 & $* * *$ & 40.0 \\
\hline 2. Legislación nacional o regional & 14.8 & 30.4 & ns & 7.7 & 21.4 & 7.1 & 60.0 & $* * a$ & 22.0 \\
\hline
\end{tabular}

$\mathrm{BAC}=$ bachillerato, $\mathrm{ESO}=$ educación secundaria obligatoria, 1,2 y $4=$ años del nivel educativo correspondiente. $*$ Significativo con $\mathrm{p} \leq 0.05$, ** significativo con $\mathrm{p} \leq 0.01$, *** significativo con $\mathrm{p} \leq 0.001$. ns $=$ no significativo.

${ }^{a}=\geq 25 \%$ de las casillas poseen una frecuencia esperada inferior a 5 .

Fuente: Elaborada por los autores.

A modo de ejemplo para el primer curso de la ESO, el manual ESO1A (p. 215) desarrolla el tema de las zonas ZEPA para una Comunidad particular: "En la Comunidad Valenciana hay 18 ZEPA, con una superficie total de 268666 ha, un 11.6\% del territorio...”. Ya en el cuarto curso de la ESO se amplía la información sobre las estrategias de conservación: “*Parques Nacionales [...]. *Reservas integrales de interés científico [...]. *Parques naturales [...]. *Parajes naturales de interés nacional. [...] [Con] la promulgación, en 1916, de la Ley General de Parques Nacionales, son parques nacionales [...]” (ESO4E, pp. 224-225). 
A diferencia de lo encontrado para la sub-categoría creación de reservas y parques, el comercio de flora y fauna silvestres tuvo una escasa representación en los manuales de ambos niveles educativos, no superando el $30 \%$ de presencia (Tabla 2). En este apartado adquiere interés la Convención sobre el Comercio Internacional de Especies de Fauna y Flora Amenazadas o CITES (por su sigla en inglés, Convention on International Trade in Endangered Species of Wild Fauna and Flora), que nace en 1973 como un acuerdo entre los miembros de la Unión Internacional para la Conservación de la Naturaleza o IUCN (del inglés International Union for Conservation of Nature). El principal objetivo de la convención CITES es garantizar la supervivencia de las especies silvestres en su medio natural por medio del establecimiento de distintos grados de protección, según la vulnerabilidad de las especies. En el presente estudio, un $60 \%$ de los libros del segundo curso del bachillerato trató el tema, frente a un $15 \%$ de los manuales de la ESO (Tabla 2). El mayor desarrollo temático, como en casos anteriores, tuvo lugar en el segundo curso del bachillerato:

Una herramienta fundamental para este tipo de conservación, y también para muchos casos de conservación "in situ", son las listas rojas de la UICN [...]. Esta organización, fundada en 1948, es pionera en la conservación de la biodiversidad [...]. A ella se debe el concepto de "lista roja" propuesto a finales de los años cincuenta del siglo XX, en la época en que la biodiversidad era sinónimo de diversidad de especies [...] (BAC2A, p. 99).

\section{iii) Aspectos legales}

En relación con los aspectos legales de la conservación de la biodiversidad, los tratados y cumbres internacionales tuvieron mayor cobertura que la legislación nacional o regional en ambos niveles educativos (Tabla 2). Los manuales del segundo curso del bachillerato fueron los que más desarrollaron estas temáticas, mientras que solo un manual del primer curso de la ESO trató el marco legal de la protección animal: “[...] el Departamento de Agricultura, Ganadería y Pesca elaboró una ley [...] [por] la cual se prohíben la caza, la captura, la importación, la exportación, la venta y exhibición pública de las especies en peligro de extinción” (ESO1M, p. 76). En los manuales del cuarto curso de la ESO encontramos referencias a las reservas de la Comunidad Valenciana, como por ejemplo: "La albufera. Fue la primera zona natural declarada espacio protegido en nuestra comunidad, hecho que se produjo en 1986" (ESO4D, p. 94). Por último, para el segundo curso del bachillerato se destaca la detallada cobertura de tratados y legislaciones que presenta cronológicamente el manual BAC2J (p. 391):

En España en el año 1916 se promulgó la Ley General de Parques Nacionales y en al año 1918 se declaran los dos primeros Parques Nacionales [...]. En 1975 se aprueba la Ley de Espacios Naturales Protegidos [...]. A raíz de ella se crea una red de once Parques Naturales y ocho Parajes Naturales. En 1989 se establece la Ley de Conservación de los Espacios Naturales y de la Fauna y Flora silvestres [...] Así se modifica la Ley de Conservación de Espacios Naturales [...] 


\section{c) Niveles de complejidad en el tratamiento temático}

Se realizó un análisis de componentes principales (ACP) para determinar cuáles son los factores o conjunto de variables (números romanos en azul, Figura 1) más importantes en la diferenciación de los manuales de la ESO y del bachillerato (puntos negros, Figura 1). Se encontró que las dos primeras componentes acumularon una proporción de varianza correspondiente al $50.1 \%$, con autovalores de 4.06, 2.19 y 1.27 para los componentes 1,2 y 3 , respectivamente. Además, la mayoría de las categorías de análisis presentadas en la Tabla 1 y 2 se distribuyeron en la componente 1 , indicando la importancia de las categorías y subcategorías definidas en la primera etapa de la presente investigación. Sin embargo, los ángulos entre las variables indican que las causas de la existencia de la biodiversidad (I.1 a I.5) no se relacionaron con el tratamiento de las estrategias para su conservación (variables II.1 a IV.1), ya que éstas tienen un mayor autovector en el componente 2. Por otro lado, el análisis de conglomerados (“clúster") (margen izquierdo superior - en verde -, Figura 1), muestra que los manuales del primer curso de la ESO y del bachillerato fueron los más similares, seguido por los textos del cuarto curso de la ESO. Teniendo en cuenta los resultados de este apartado se definen tres niveles de complejidad para el tratamiento de las variables estudiadas: (a) la mayoría de los libros de segundo curso del bachillerato se ubicó en el margen derecho de la Figura 1, lo que representó el nivel más complejo en el tratamiento de las causas de la existencia y estrategias de conservación de la biodiversidad; (b) un nivel intermedio de complejidad fue definido por un conjunto de libros de los tres cursos restantes, aunque con preponderancia de manuales del cuarto curso de la ESO; y (c) el nivel más bajo de complejidad quedó integrado por manuales de los primeros cursos de la ESO y del bachillerato (margen izquierdo de la Figura 1).

Figura 1. Análisis de componentes principales para las categorías y subcategorías de estudio (azul) y análisis de conglomerados por ligamiento promedio y distancia euclídea (verde) para manuales de la educación secundaria española

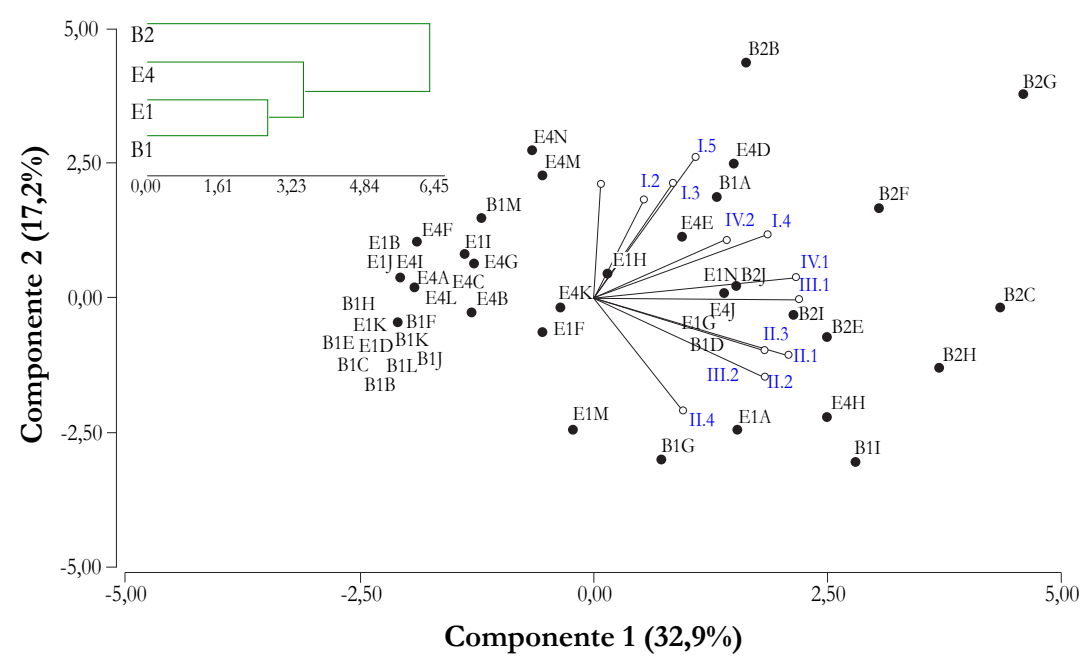

Los porcentajes de varianza explicada se dan entre paréntesis. E= ESO, B =bachillerato. Referencias en las Tablas 1 y 2, y en el Anexo I.

Fuente: Elaborado por los autores. 


\section{Conclusiones e implicancias educativas}

Los resultados de este estudio indican que las ideas de climax y equilibrio ecológico desarrolladas en los manuales escolares actúan como obstáculo para comprender los disturbios como causas de la existencia de la biodiversidad debido a que: (a) los ejemplos de sucesiones de la mayoría de los libros culminaron en comunidades boscosas (incluso cuando partieron de ecosistemas lacustres); (b) la diversidad de especies se asoció a la complejidad ecosistémica, la que indefectiblemente aumentó con la riqueza (y ésta con el paso del tiempo); (c) las sucesiones se ejemplificaron con poblaciones vegetales, sin otros organismos, y a que (d) en el estado de clímax las comunidades carecían de especies dominantes.

Por otro lado, se cuestiona que las razones estéticas, biológicas y éticas estuvieran presentes en un porcentaje menor al $20 \%$ de los manuales estudiados, salvo en el caso del segundo curso del bachillerato. Se evidencia que los manuales escolares pueden presentar a la conservación de la biodiversidad como un "dogma", sin que se fundamenten las motivaciones que llevan a acciones como la creación de reservas, de parques nacionales o la firma de tratados internacionales. Como consecuencia, la enseñanza se vuelve monumentalista (CHEVALLARD, 2004), pues deja de cuestionar el contenido y prioriza el estudio "porque sí” de los saberes. En otros casos, las razones de la conservación de la biodiversidad adquirieron connotaciones biologicistas y utilitaristas, abandonando el punto de vista bioético (SILVA; KRASILCHIK, 2013) y descuidando las dimensiones epistemológicas, sociales y culturales de la biodiversidad (GARCÍA GÓMEZ; MARTÍNEZ BERNAT, 2010; GUTIÉRREZ HÉRNANDEZ, 2013). Esta tendencia se plasmó incluso en la interpretación de los bienes y servicios que aporta la biodiversidad, ya que la recreación, la educación e inspiración, y los valores espirituales y religiosos estuvieron prácticamente ausentes en los mismos textos (BERMUDEZ et al.) ${ }^{6}$. En este contexto, se plantea la necesidad de enseñar la biodiversidad como un proceso en donde los saberes no constituyan monumentos que el profesor expone a los estudiantes, sino como un conjunto de constructos conceptuales, procedimentales y axiológicos que se ponen en juego para resolver problemáticas de la vida cotidiana. De otro modo, como señalan Otero, Fanaro y Corica (2013), se instala en la enseñanza un proceso sistemático de introducción de respuestas a preguntas ocultas o inexistentes, en donde los alumnos dejan de reconocer el origen, la utilidad, la razón de ser, el porqué y el para qué del saber enseñado.

Por otro lado se cuestiona que los libros del primer curso del bachillerato desarrollen escasamente las estrategias y tratados de conservación cuando forman parte del listado de contenidos de la materia "Biología y Geología", específicamente en el ítem "los animales en peligro de extinción. Acciones para la conservación de la diversidad” (ESPAÑA, 2007a, p. 45435). Las razones para conservar la biodiversidad que ofrecen los manuales españoles ponen en evidencia el proceso de desincretización señalado por Verret (1975), por el cual un saber se parcela en compartimientos escindidos de las problemáticas que les dan origen. A su vez, el tratamiento de las cuestiones legales revela una desconexión de los libros de texto con la legislación española y de las comunidades autónomas del entorno cercano de los estudiantes.

\footnotetext{
${ }^{6}$ Datos no publicados.
} 
El análisis de componentes principales permite concluir que el desarrollo de las causas de la biodiversidad, como son la latitud, el régimen de disturbios y el clima, fue independiente del tratamiento que recibieron las estrategias para su conservación, como la creación de parques nacionales o el establecimiento de convenios que regulen el comercio de especies en peligro de extinción. Este desligamiento entre el ámbito de los valores (normas) y el cognitivo, con fuerte impronta del contenido biológico y geológico, puede representar un obstáculo para el desarrollo de aprendizajes y actitudes ambientalmente favorables. Se suma a ello el escaso grado de complejización en el tratamiento temático de los manuales que fuera observado desde el primer curso de la ESO hasta el primero del bachillerato.

A partir de este estudio se recomiendan los manuales escolares ubicados en el margen derecho de la Figura 1 para trabajar con los alumnos las causas de la diversidad biológica y las estrategias para su conservación. En este sector se ubican libros correspondientes a tres de los cuatro cursos estudiados, por lo que una cuidadosa vigilancia epistemológica a los mismos garantizaría una adecuada transposición para la educación secundaria española. En particular, se sugiere el empleo del manual BAC2B para el estudio y adecuación a otros cursos de las causas de la existencia de la biodiversidad, y del texto BAC2I para las estrategias de su conservación. Asimismo, los manuales BAC2G, BAC2C y BAC2H podrán orientar al docente en la transposición de la totalidad de categorías contempladas en este estudio.

En la noosfera, el trabajo didáctico de la selección y organización del contenido de biodiversidad exige al docente una transposición holística, que adapte los saberes sabios y de expertos a las necesidades e intereses del nivel educativo y contexto particular de enseñanza. Para ello, el profesor de Biología debe contemplar criterios lógicos, psicológicos y socio-culturales, tomando decisiones sobre el enfoque y grado de complejidad del conocimiento a enseñar. En este sentido, se vuelve necesario establecer el alcance para el contenido establecido en los diseños curriculares, seleccionar y analizar cuidadosamente la información contenida en los manuales escolares (PERALES PALACIOS; VÍLCHEZ GONZÁLEZ, 2012). Ello convierte al docente en un mediador calificado en la construcción del conocimiento escolar e incorpora la vigilancia epistemológica a las competencias y saberes profesionales. Tomando en consideración esta situación, sería interesante continuar la línea de investigación registrando las estrategias que utilizan los docentes en sus aulas para enseñar sobre la biodiversidad y legitimar el conocimiento, y buscando responder qué relación guardan estas actividades con las propuestas para los estudiantes en los libros de texto y, al mismo tiempo, qué manuales emplean para su estudio y capacitación profesional. 


\section{Referencias}

ALMEIDA, A. V. D.; FALCÃO, J. T. D. R.; ROCHA, J. T. D. As teorias de Lamarck e Darwin nos livros didáticos de biologia no Brasil. Ciência \& Educação, Bauru, v. 16, n. 3, p. 649-665, 2010. Disponible en: <http://dx.doi.org/10.1590/S1516-73132010000300010>. Acceso el: 21 mayo 2015.

ALMEIDA, P.; FIGUEIREDO, O.; GALVÃO, C. A argumentação em tarefas de manuais escolares portugueses de biologia e de geologia. Investigações em Ensino de Ciências, Porto Alegre, v. 17, n. 3, p. 571-591, 2012.

ASSIS, S. S.; PIMENTA, D. N.; SCHALL, V. T. A dengue nos livros didáticos de ciências e biologia indicados pelo Programa Nacional do Livro Didático. Ciência \& Educação, Bauru, v. 19, n. 3, p. 633-656, 2013. Disponible en: <http://dx.doi.org/10.1590/S1516$73132013000300009>$. Acceso el: 21 mayo 2015.

BALZARINI, M. G. et al. Infostat: manual del usuario. Córdoba: Editorial Brujas, 2008. BERMUDEZ, G. M. A.; DE LONGHI, A. L. Análisis de la transposición didáctica del concepto de biodiversidad: orientaciones para su enseñanza. In: MOLINA, A.; MARTÍNEZ, C. A.; GALLEGO, O. (Org.). Algunas problemáticas de investigación en la enseñanza de las ciencias naturales en América Latina. Bogotá: Universidad Distrital Francisco José de Caldas, 2012. p. 115-153.

BERMUDEZ, G. M. A. et al. La transposición del concepto de diversidad biológica: un estudio sobre los libros de texto de la educación secundaria española. Enseñanza de las Ciencias, Barcelona, v. 32, n. 3, p. 285-302, 2014.

BOSCH, M.; GASCÓN, J. 25 años de transposición didáctica. In: RUIZ-HIGUERAS, L.; ESTEPA, A.; GARCÍA, F. J. (Org.). Sociedad, escuela y matemáticas: aportaciones de la teoría antropológica de lo didáctico. Jaén: Servicio de Publicaciones de la Universidad de Jaén, 2006. p. 385-406.

CAMPOS, A. F.; LIMA, E. N. Ciclo do nitrogênio: abordagem em livros didáticos de ciências do ensino fundamental. Investigações em Ensino de Ciências, Porto Alegre, v. 13, n. 1, p. 35-44, 2008. Disponible en: < http://www.if.ufrgs.br/ienci/artigos/Artigo_ID23/ v13_n1_a2008.pdf >. Acceso el: 21 maio 2015.

CARDOSO-SILVA, C. B.; OLIVEIRA, A. C. Como os livros didáticos de biologia abordam as diferentes formas de estimar a biodiversidade? Ciência \& Educação, Bauru, v. 19, n. 1, p. 169-180, 2013. Disponible en: <http://dx.doi.org/10.1590/S1516-73132013000100012>. Acceso el: 21 mayo 2015.

CHEVALLARD, Y. La transposición didáctica: del saber sabio al saber enseñado. Buenos Aires: Aique, 1991.

. Vers une didactique de la codisciplinarité: notes sur une nouvelle épistemologie scolaire. In: JOURNÉES DE DIDACTIQUE COMPARÉE, 2004, Lyon. Disponible en: $<$ http:/ /yves.chevallard.free.fr/spip/spip/article.php3?id_article=45>. Acceso el: 10 marzo 2014. 
Bermudez, G. M. A.; De Longhi, A. L.; Gavidia, V.

CONVENTION ON BIOLOGICAL DIVERSITY. Interlinkages between biological diversity and climate change: advice on the integration of biodiversity considerations into the implementation of the United Nations Framework Convention on Climate Change and its Kyoto protocol. Montreal: CBD, 2003. (CBD Technical series, 10). Disponible en: <http://www.rac-spa.org/node/480>. Acceso el: 21 mayo 2015.

Spain country profile. Biodiversity facts. Disponible en: <http://www.cbd.int/ countries/profile/default.shtml?country=es\#status>. Acceso el: 22 mayo 2015.

Text of the CBD. 1992. Disponible en: < http://www.cbd.int/convention/text/>. Acceso el: 14 dic. 2013.

COUNCIL OF THE EUROPEAN COMMUNITIES. Council directive of 2 April 1979 on the conservation of wild birds (79/409/EEC). Disponble en: <http://eur-lex.europa. eu/legal-content/EN/TXT/PDF/?uri=CELEX:31979L0409\&from=ES>. Acceso el: 22 mayo 2015.

ESPAÑA. Real decreto 1007/1991, de 14 de junio, por el que se establecen las enseñanzas mínimas correspondientes a la educación secundaria obligatoria. Boletín Oficial del Estado, Madrid, n. 152, 26 jun. 1991. Disponible en: < http://noticias.juridicas.com/base_ datos/Derogadas/r0-rd1007-1991.html>. Acceso el: 21 mayo 2015.

Real decreto 1467/2007, de 2 de noviembre, por el que se establece la estructura del bachillerato y se fijan sus enseñanzas mínimas. Boletín Oficial del Estado, Madrid, n. 266, p. 45381-45477, 6 nov. 2007a. Disponible en: <http://www.boe.es/boe/dias/2007/11/06/ pdfs/A45381-45477.pdf > . Acceso el: 21 mayo 2015.

Real decreto 1631/2006, de 29 de diciembre, por el que se establecen las enseñanzas mínimas correspondientes a la educación secundaria obligatoria. Boletín Oficial del Estado, Madrid, n. 5, p. 677-773, 05 ene. 2007b. Disponible en: <http://www.boe.es/boe/ dias/2007/01/05/pdfs/A00677-00773.pdf>. Acceso el: 21 mayo 2015.

EUROPEAN COMMISSION. The birds directive. Disponible en: < http://ec.europa.eu/ environment/nature/legislation/birdsdirective/index_en.htm>. Acceso el: 05 marzo 2014.

EUROPEAN PARLIAMENT. Our life insurance, our natural capital: an EU biodiversity strategy to 2020. [Brussels: European Commission, 2011]. Disponible en: <http:/ / ec.europa. $\mathrm{eu} /$ environment/nature/biodiversity/comm2006/pdf/EP_resolution_april2012.pdf>.

Visitado el: 21 mayo 2015.

GARCÍA GÓMEZ, J.; MARTÍNEZ BERNAT, F. J. Cómo y qué enseñar de la biodiversidad en la alfabetización científica. Enseñanza de las Ciencias, Barcelona, v. 28, n. 2, p. 175-174, 2010.

GUTIÉRREZ HÉRNANDEZ, S. G. Aspectos históricos y epistemológicos del concepto biodiversidad. Bio-grafía: escritos sobre biología y su enseñanza, Bogotá, v. 6, n. 10, p. 84-93, 2013. 
IBARRA MURILLO, J.; GIL QUÍLEZ, M. J. Enseñar los cambios ecológicos en la secundaria: un reto en la transposición didáctica. Enseñanza de las Ciencias, Barcelona, v. 23, n. 3, p. 345-356, 2005.

MALDONADO GONZÁLEZ, F; GONZÁLEZ GARCÍA, F; JIMÉNEZ TEJADA, M. P. Las ilustraciones de los ciclos biogeoquímicos del carbono y nitrógeno en los textos de secundaria. Revista Eureka sobre Enseñanza y Divulgación de las Ciencias, Cádiz, v. 3, n. 4, p. 442-460, 2007.

MARTÍNEZ BERNAT, F. J.; GARCÍA GÓMEZ, J. Análisis del tratamiento didáctico de la biodiversidad en los libros de texto de biología y geología en secundaria. Didáctica de las

Ciencias Experimentales y Sociales, Valencia, n. 23, p. 109-122, 2009.

MARTINEZ-GRACIA, M. V.; GIL-QUÍLEZ, M. J.; OSADA, J. Analysis of molecular genetics content in Spanish secondary school textbooks. Journal of Biological Education, Philadelphia, v. 40, n. 2, p. 53-60, 2006.

MENZEL, S.; BÖGEHOLZ, S. The loss of biodiversity as a challenge for sustainable development: how do pupils in Chile and Germany perceive dilemmas? Research in Science Education, Dordrecht, v. 39, n. 4, p. 429-447, 2009.

OTERO, M. R.; FANARO, M. A.; CORICA, A. R. La teoría antropológica de lo didáctico en el aula de matemática. Buenos Aires: Dunken, 2013.

PERALES PALACIOS, F. J.; VÍLCHEZ GONZÁLEZ, J. M. Libros de texto: ni contigo ni sin ti tienen mis males remedio. Alambique: didáctica de las ciencias experimentales, Barcelona, n. 70, p. 75-82, 2012.

RODRÍGUEZ MIRANDA, F. D. P. et al. El conocimiento escolar sobre los animales y las plantas en primaria: Un análisis del contenido específico en los libros de texto. Revista Electrónica de Enseñanza de las Ciencias, Vigo, v. 13, n. 1, p. 97-114, 2014.

SELLES, S. S.; FERREIRA, M. S. Analyzing experimentation in Brazilian science textbooks from a socio-historical approach. Journal of Science Education, Bogotá, v. 11, n. 1, 44-47, 2010.

SILVA, P. F.; KRASILCHIK, M. Bioética e ensino de ciências: o tratamento de temas controversos - dificuldades presentadas por futuros professores de ciências e de biologia. Ciência \& Educação, Bauru, v. 19, n. 2, p. 379-392, 2013. Disponible en: < http://dx.doi. org/10.1590/S1516-73132013000200010>. Acceso el: 22 mayo 2015.

VERRET, M. Le temps des études. Paris: Honoré Champion, 1975. 
Bermudez, G. M. A.; De Longhi, A. L.; Gavidia, V.

Anexo A. Listado y códigos de identificación de los manuales escolares españoles analizados

a. Primer curso de la ESO: ciencias de la naturaleza

\begin{tabular}{|l|l|l|}
\hline Código & \multicolumn{1}{|c|}{ Autor } & \multicolumn{1}{c|}{ Imprenta } \\
\hline ESO1A & FERNÁNDEZ ESTEBAN, M. Á. et al. & $\begin{array}{l}\text { Valencia: Vincens Vives Educació } \\
\text { Secundària, 2008 }\end{array}$ \\
\hline ESO1B & GARCÍA GREGORIO, M. et al. & Paterna: Ecir, 1996. \\
\hline ESO1C & LÓPEZ FENOY, V. et al. & Zaragoza: Edelvives, 1996. \\
\hline ESO1D & PEDRINACI, E. L. C. et al. & Madrid: Ediciones SM, 2003 \\
\hline ESO1E & BALIBREA, S. et al. & Valencia: Anaya, 2007 \\
\hline ESO1F & BARRIO GÓMEZ DE AGÜERO, J. et al. & Madrid: Oxford Educación, 2007 \\
\hline ESO1G & CASAS ÚBEDA, J. M. et al. & Barcelona: Santillana, 2004 \\
\hline ESO1H & CARRIÓN, F. et al. & Barcelona: Anaya, 1996 \\
\hline ESO1I & BRINCONES, I. & Valencia: Voramar Santillana, 1996 \\
\hline ESO1J & BARRIO GÓMEZ DE AGÜERO, J. et al. & Madrid: Oxford Educación, 2002 \\
\hline ESO1K & DEL CARMEN, L. et al. & Toledo: Ediciones SM, 1997 \\
\hline ESO1L & PANADERO CUARTERO, J. E. et al. & Madrid: Bruño, 1996 \\
\hline ESO1M & SÁNCHEZ, D. et al. & Barcelona: Santillana, 2000 \\
\hline
\end{tabular}

b. Cuarto curso de la ESO: Biología y Geología

\begin{tabular}{|l|l|l|}
\hline Código & \multicolumn{1}{|c|}{ Autor } & \multicolumn{1}{c|}{ Imprenta } \\
\hline ESO4A & GARCÍA, C.; RIAZA MOYA, C. & Madrid: Bruño, 1995 \\
\hline ESO4B & $\begin{array}{l}\text { ELIZALDE GÓMEZ, J. R.; SÁNCHEZ } \\
\text { DELGADO, I. }\end{array}$ & Madrid: McGraw-Hill, 1995 \\
\hline ESO4C & FLORES SARRIÓN, M. J.; GOMEZ GOMEZ, S. & Madrid: Akal, 1998 \\
\hline ESO4D & BALIBREA, S. et al. & Madrid: SM, 2003 \\
\hline ESO4E & ALBALADEJO MARCET, C. et al. & Estella: Oxford, 2003 \\
\hline ESO4F & BERGES, T. et al. & Madrid: Anaya, 1998 \\
\hline ESO4G & GARCÍA GREGORIO, M. et al. & Paterna: ECIR, 2003 \\
\hline ESO4H & PANADERO CUARTERO, J. E. et al. & Madrid: Bruño, 1999 \\
\hline ESO4I & BELART RODRÍGUEZ, A. et al. & Madrid. EDITEX, 1998 \\
\hline ESO4J & GARCÍA GREGORIO, M. et al. & Paterna: ECIR, 2008 \\
\hline ESO4K & BRUSI, D. et al. & Barcelona: Voramar Santillana, 2003 \\
\hline ESO4L & GARCÍA GREGORIO, M. et al. & Paterna: ECIR, 1999 \\
\hline ESO4M & GARCÍA GIMÉNEZ, L. et al. & Valencia: Tilde, 1999 \\
\hline ESO4N & PEDRINACI, E.; GIL, C. & Madrid: SM, 2003 \\
\hline
\end{tabular}


c. Primer curso del Bachillerato: Biología y Geología (se especifica si es distinto)

\begin{tabular}{|l|l|l|l|}
\hline Código & \multicolumn{1}{|c|}{ Autor } & \multicolumn{1}{c|}{ Título } & \multicolumn{1}{c|}{ Imprenta } \\
\hline BAC1A & PULIDO, C. et al. & & Madrid: Anaya, 2002 \\
\hline BAC1B & GARCÍA GREGORIO, M. et al. & & Valencia: ECIR, 2003 \\
\hline BAC1C & PULIDO, C. et al. & & Madrid: Anaya, 2000 \\
\hline BAC1D & FERRER MARI, N. et al. & & Madrid: Anaya, 2008 \\
\hline BAC1E & FURIÓ EGEA, J. et al. & & Valencia: ECIR, 1997 \\
\hline BAC1F & ESCARRÉ ESTEVE, A. et al. & & Madrid: Santillana, 1996 \\
\hline BAC1G & CABRERIZO RIBATE, B. et al. & & $\begin{array}{l}\text { Madrid: Oxford University } \\
\text { Press, 2002 }\end{array}$ \\
\hline BAC1H & JIMENO, A. et al. & & Madrid: Santillana, 2002 \\
\hline BAC1I & ANGUITA, F. et al. & & Madrid: SM, 1997 \\
\hline BAC1J & ANGLADA ESQUIUS, M. et al. & & Barcelona: Edebé, 1998 \\
\hline BAC1K & BARUTELL ENCINA, A. et al. & $\begin{array}{l}\text { Ciencias naturales: } \\
\text { bachillerato 1 }\end{array}$ & Barcelona: Anaya, 1998 \\
\hline BAC1L & ARIAS, M. et al. & Biologia 1 & Barcelona: Castellnou, 1997 \\
\hline BAC1M & PEDRINACI, E. et al. & $\begin{array}{l}\text { Ciencias de la } \\
\text { naturaleza y de la salud }\end{array}$ & Toledo: SM, 2002 \\
\hline
\end{tabular}

d. Segundo curso del Bachillerato: Ciencias de la Tierra y medioambientales

\begin{tabular}{|l|l|l|}
\hline Código & \multicolumn{1}{|c|}{ Autor } & \multicolumn{1}{c|}{ Imprenta } \\
\hline BAC2A & LÓPEZ, T. & Madrid: Santillana, 2003 \\
\hline BAC2B & ASENSI MARQUÉS, J. J. et al. & Valencia: ECIR, 1998 \\
\hline BAC2C & CABRERA, M. E. et al. & Madrid: Editex, 1996 \\
\hline BAC2D & GURRERA, M. A. et al. & Barcelona: Edebé, 2009 \\
\hline BAC2E & GURRERA, M. A. et al. & Barcelona: Edebé, 2003 \\
\hline BAC2F & ALFONSO CERVEL, F. et al. & Estella: Oxford Educación, 2006 \\
\hline BAC2G & GARCÍA GREGORIO, M. et al. & Paterna: ECIR, 2004 \\
\hline BAC2H & RUBIO SÁEZ, N. et al. & Sabadell: Anaya, 2003 \\
\hline BAC2I & DIODORA CALVO, M. T. et al. & Madrid: McGraw-Hill, 2005 \\
\hline BAC2J & DIODORA CALVO, M. T.; SALVACHÚA & Madrid: McGraw-Hill, 2001 \\
& RODRÍGUEZ, J. & \\
\hline
\end{tabular}

